\title{
Automated enclosure and protection system for compact solar-tracking spectrometers
}

\author{
Ludwig Heinle and Jia Chen \\ Environmental Sensing and Modeling, Department of Electrical and Computer Engineering, \\ Technische Universität München, 80333 Munich, Germany \\ Correspondence: Jia Chen (jia.chen@tum.de)
}

Received: 12 August 2017 - Discussion started: 24 August 2017

Revised: 7 February 2018 - Accepted: 9 February 2018 - Published: 17 April 2018

\begin{abstract}
A novel automated enclosure for protecting solartracking atmospheric instruments was designed, constructed, and successfully tested under various weather conditions. A complete automated measurement system, consisting of a compact solar-tracking Fourier transform infrared (FTIR) spectrometer (EM27/SUN) and the enclosure, has been deployed in central Munich to monitor greenhouse gases since 2016 and withstood all critical weather conditions, including rain, storms, and snow. It provided ground-based measurements of column-averaged concentrations of $\mathrm{CO}_{2}, \mathrm{CH}_{4}, \mathrm{O}_{2}$, and $\mathrm{H}_{2} \mathrm{O}$ throughout this time.

The enclosure protects the instrument from harmful environmental influences while allowing open-path measurements in sunny weather. The newly developed and patented cover, a key component of the enclosure, permits unblocked solar measurements while reliably protecting the instrument. This enables dynamic decision regarding taking measurements, and thus increases the number of data samples. This enclosure leads to a fully automated measurement system, which collects data whenever possible without any human interaction. In the long term, the enclosure will provide the foundation for a permanent greenhouse gas monitoring sensor network.
\end{abstract}

\section{Introduction}

Anthropogenic greenhouse gas (GHG) emissions into the atmosphere have risen to a worrying level over the last few decades. There is little doubt that this impacts the climate on Earth and eventually the well-being of humanity. To understand greenhouse gas sources, sinks, and transportation, re- liable and precise atmospheric concentration measurements are required. State-of-the-art ground-based and spaceborne spectrometers are used to measure column-averaged gas concentrations by analyzing the gas absorptions for specific frequencies of sunlight. Ground-based solar-viewing spectrometers use the sun as a light source. Gas molecules, such as $\mathrm{O}_{2}$, $\mathrm{CO}_{2}$, and $\mathrm{CH}_{4}$, interact with the sunlight on its path through the atmosphere, resulting in absorption lines in the recorded sun spectrum. By observing the intensity attenuation of light at specific frequencies, the concentration of gas molecules within the air column can be determined.

The Total Carbon Column Observing Network (TCCON; Wunch et al., 2011) is a global network that measures total column concentrations. The network contains Bruker IFS $125 \mathrm{HR}$ spectrometers, which provide accurate data to validate satellite observations. However, these instruments are large (close to $2 \times 3 \mathrm{~m}$ footprint), weigh more than half a ton and are expensive (Bruker Optic GmbH, 2006). Further, their operation is costly in terms of staffing. Therefore, many working groups operating $125 \mathrm{HR}$ spectrometers use remote control or automated systems. A team at the Belgian Institute for Space Aeronomy developed the Bruker Automation and Remote COntrol System (BARCOS; Neefs et al., 2007). Later, Geibel et al. (2010) developed an automated system to deploy the $125 \mathrm{HR}$ on Ascension Island. The main goal of these systems is to lower the operational costs by reducing the need for local human interaction for operation. However, operation of a $125 \mathrm{HR}$ still requires regular skilled onsite maintenance, due to the degradation of interferometric alignment over a timescale of months (Hase, 2012).

A portable solar-tracking Fourier transform infrared (FTIR) spectrometer, the EM27/SUN (Gisi et al., 2011, 
2012; Hase et al., 2016), has been employed for many applications in urban greenhouse gas emissions studies in recent years. It is lightweight, compact, and very robust, and it has attained a comparable precision to TCCON instruments (Frey et al., 2015; Hedelius et al., 2016; Chen et al., 2016). It is easy to transport and operate, and therefore permits more flexible site selection. While the large 125HR units are deployed for global observation, the small EM27/SUN units are more often utilized for local and city source investigations (Hase et al., 2015; Chen et al., 2016, 2017, 2018; Franklin et al., 2017; Viatte et al., 2017; Toja-Silva et al., 2017). Further, it has been deployed on a ship (Klappenbach et al., 2015) and in a mobile observatory to assess volcanic emissions (Butz et al., 2017). The EM27/SUN is equipped with a solar-tracking unit, consisting of motorized rotating mirrors to direct the light into the spectrometer. An external laptop is connected to the spectrometer via multiple connectors. Even though the EM27/SUN body itself is weather resistant, the other components, e.g., the laptop, the control electronics, the solar tracker, and especially the tracker mirrors, need to be protected. Hedelius et al. (2016) found degradation of the solar tracker mirrors in their spectrometer, which could have been caused by the combined action of reactive substances, sea-salt aerosols, and humidity above saturation, in which case the contaminated water droplets might be deposited on the mirrors.

Much human effort is necessary to operate the instrument at a measurement site. A person needs to set up the system every time before measuring. This setup needs to be manually dismantled and stored safely, whenever the weather changes to rainy or stormy conditions. Besides wear and tear of the connectors, much costly labor is necessary. Even though the operators do not need to be highly trained, their permanent attention is required. Consequently, the very limited availability for measuring during short periods of good conditions reduces the amount of data collected.

We have developed an automated enclosure (Fig. 1) that houses and protects the measurement system, while allowing measurements during good weather conditions. It eliminates the need to dismantle the system after each measurement period, and protects against harmful weather conditions. A remote operator can monitor multiple stations simultaneously, while the setup and dismantling times can be reduced to a minimum. The easy handling combined with the small size and low power consumption reduce operational costs of such a measurement system to nearly zero.

To construct such an enclosure, several challenges must be faced. A safe and reliable protection against environmental influences, like dirt, thunderstorms, and even hail, is needed for the measurement system, though it is necessary for the solar-tracking mirrors of the instruments to be directly exposed to the sunlight during sunny weather conditions. Therefore, the prime directive for the enclosure design is reliable protection and maximized amount of measurement data. Other criteria include the best possible remote control-

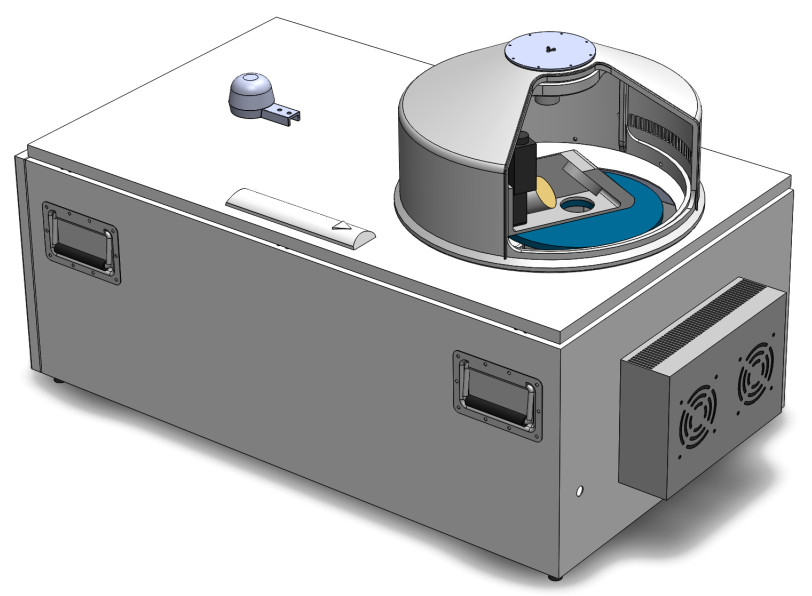

Figure 1. 3-D computer-aided design (CAD) model of the enclosure including the base cabinet, the cover, the thermal electrical cooler, and the rain sensor. These hardware components are explained in Sect. 2.

lability via the internet, the thermal stability of the system, and a stable and uninterrupted power supply. The latter ensures error-free collection of the data and prevents the system from experiencing unpredictable situations. To conclude, the enclosure is designed for automatic operation without the need for human interaction.

\section{Hardware and setup}

An overview of the components of the automated enclosure is provided in Fig. 2 and its dimensions and weight are given in Table 1. These components are explained in the following subsections.

\subsection{Base cabinet}

As previously mentioned, the EM27/SUN spectrometer requires physical protection. Therefore, a stable and waterproof control cabinet is placed with its opening facing up, serving as the base cabinet for the enclosure. In this orientation, the door is located on top and allows easy access to all equipment inside. Rubber feet on the bottom ensure a slightly elevated stable position. The electrical components of the enclosure, such as power distribution and power supplies, are attached to a DIN rail. All wiring is stowed in cable ducts to keep the inside neatly organized, easy to access, and operating reliably.

The base cabinet depicted in Fig. 3 is manufactured from steel. Thus, it is very strong and durable. Four handles, one on each corner, allow easy transportation. The measurement system comfortably fits inside and is well protected by the base cabinet. The upper end of the solar tracker, which rises through an opening in the base cabinet, is the only part of the measurement system located outside. A cover, as described 


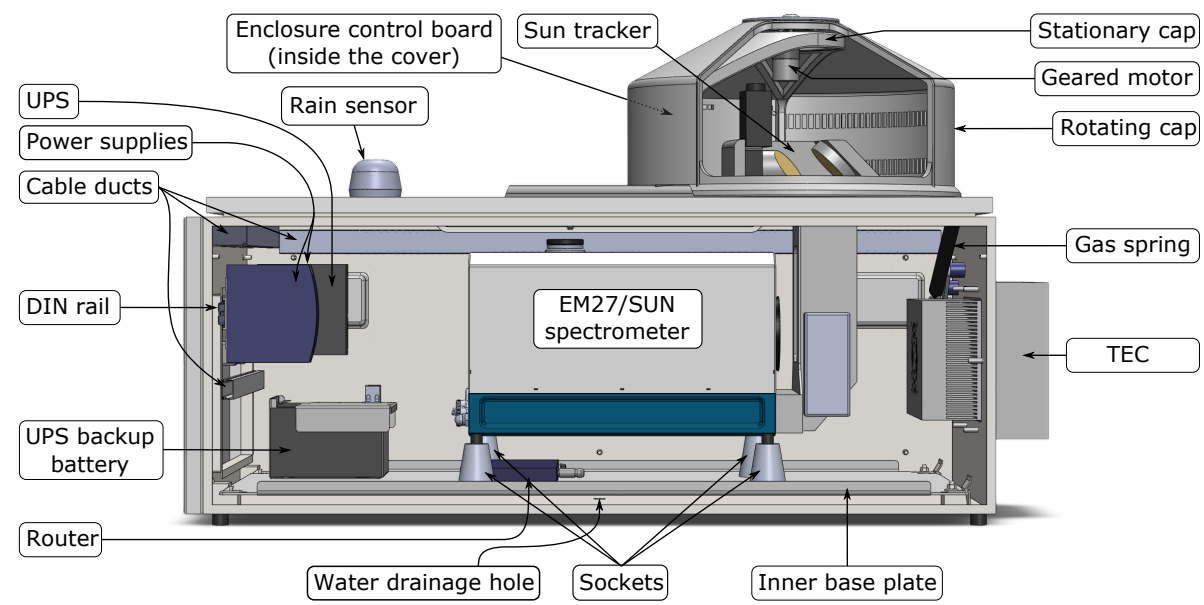

Figure 2. Enclosure overview: components and their arrangements. The laptop is placed on top of the EM27/SUN spectrometer to ensure good accessibility.

Table 1. Dimensions and weight of the enclosure.

\begin{tabular}{lll}
\hline Overall dimensions & $112 \times 62 \times 66 \mathrm{~cm}$ & (length $\times$ width $\times$ height $)$ \\
Base cabinet dimensions & $112 \times 62 \times 41 \mathrm{~cm}$ & (length $\times$ width $\times$ height) \\
Cover dimensions & $45.5 \times 25 \mathrm{~cm}$ & (diameter $\times$ height) \\
Total weight (spectrometer included) & $\approx 100 \mathrm{~kg}$ & \\
\hline
\end{tabular}

in Sect. 2.2, is mounted over the opening, covering the enclosure and protecting the instrument.

\subsection{Cover}

Because the solar tracker extends through the top of the base cabinet, it must be protected separately against harmful environmental influences. Tests with a glass dome were performed at the Karlsruhe Institute of Technology (KIT; see Sha, 2015). Unfortunately, the results were not as good as expected, since it was not possible to manufacture a dome with a sufficiently homogeneous surface. The sunlight was variously distorted depending on the solar incident angle, which affected the measurements. Moreover, dirt and dust on the glass surface and trapped humidity inside the dome could have further disturbed the measurements.

\subsubsection{Cover design}

A completely different concept is presented here. A newly developed and patented cover is mounted on top of the opening, covering the solar tracker. The cover is made of two rotationally symmetric parts with cutouts, where one fits inside the other (see Figs. 4 and 5). It protects the solar tracker from bad weather while enabling open-path measurements in dry and good weather conditions.

The outer cover rotates about the vertical symmetry axis on top of the inner cover. Its weight is carried by eight ball bearings equally distributed around the lower end of the in- ner cover. While the inner cover is mounted in a stationary position on top of the base cabinet, the outer one rotates. The rotation is driven by a simple geared electric motor, which is mounted inside the common axis of the two covers.

\subsubsection{Cover size}

The dimensions of the cover is given in Table 1. The lower size limit of the outer cover's opening is determined by the width of the mirrors. Since sunlight must not be blocked during sunny conditions, and the sun rays can be assumed to be parallel, the opening must be at least the width of the mirrors. However, in this case the cover needs to track the solar azimuth angle very precisely during the course of the day, so that it does not block the sunlight from the mirrors. Furthermore, the smaller the opening is, the more difficult the accessibility inside the cover is for any maintenance.

The upper size limit for the opening is determined by the size of the inner cover's remaining wall. During bad weather conditions or darkness, the outer cover will rotate to a closed position where the inner cover wall needs to be fully covered by the opening of the outer one. Sealing concerns and a lower demand for positioning precision led the design towards greater overlap. Finally, an opening of $90^{\circ}$ was considered to be a good trade off between overlapping and necessary tracking accuracy for the first prototype.

A rough estimation based on the 3-D CAD model shows that the permissible solar zenith angle range is $23( \pm 1)$ to $88( \pm 1)^{\circ}$. From test results (see Sect. 4.2) and the model, we 

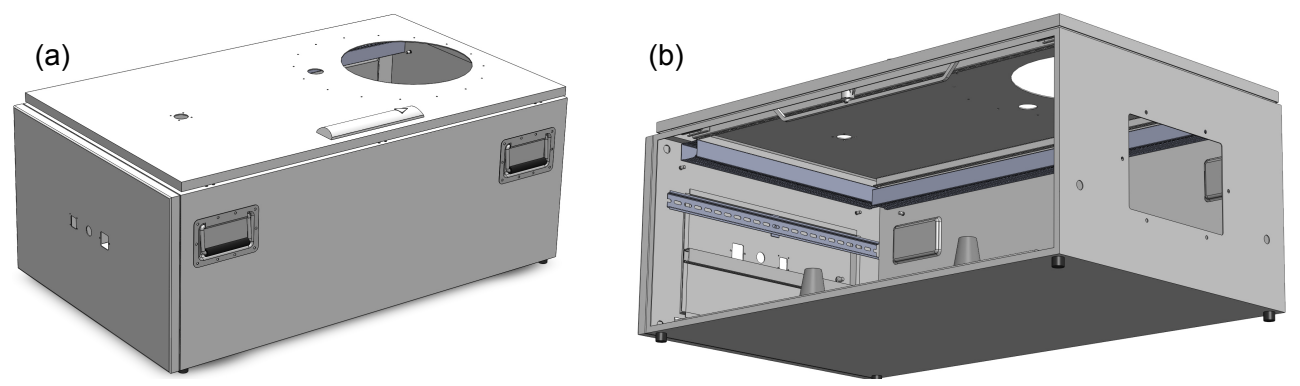

Figure 3. 3-D model of the base cabinet. In (a) the original function as a control cabinet is clearly identifiable. The handles on the side wall are mounted for easy transportation. (b) shows another perspective, with one of the side walls removed to allow a view inside the empty base cabinet.
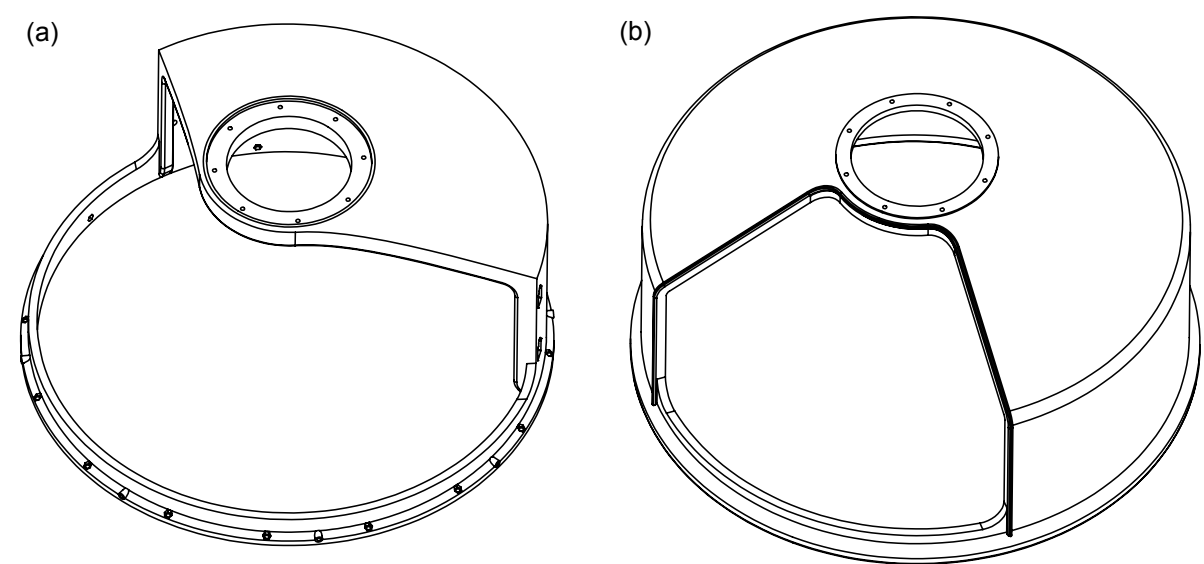

Figure 4. Drawings of the two parts of the cover. The inner cover with its large opening is depicted in (a). The cover in (b) is the outer one with a relatively small cutout.

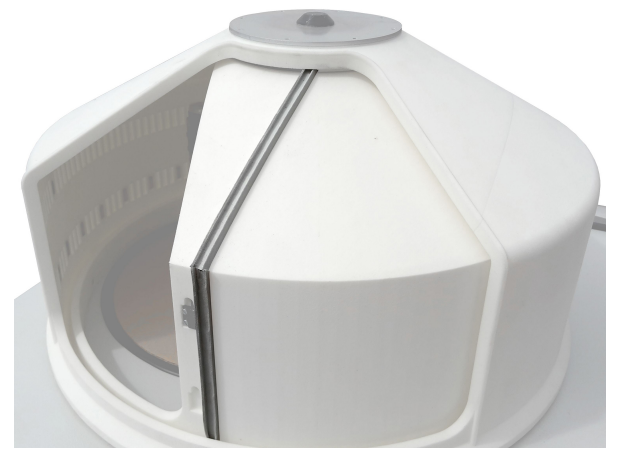

Figure 5. The image shows the gaskets that were installed to seal the gap between the two covers, preventing water from being blown into the cover by strong winds.

determined the azimuthal range is from 71 to $300^{\circ}$. The cover was designed to be deployed in Munich, so for other places on Earth, a redesign of the cutouts might be necessary. This redesign can be carried out with a simple modification of some parameters in the 3-D model, which was used to design the enclosure.

\subsubsection{Weatherproof design}

A gap between the two covers ensures a friction-free smooth rotation of the outer cover. However, rain combined with strong winds could blow water into the cover despite the overlap between the inner and outer covers. Thus, a gasket as depicted in Fig. 5 has been added to the cover. It is a window sealing strip that has been cut to the right thickness. When the cover is closed, the gasket blocks the wind and thus prevents water from entering. In addition, it fills the gap between the two covers and thus keeps insects from getting inside. Nevertheless, the rubber sealing does not obstruct the smooth rotation of the outer cover.

\subsubsection{Position determination}

The outer cover needs to track the sun for undisturbed measurements. Therefore, the actual position of the outer cover needs to be determined. To this end, we have developed an encoder using magnets and reed sensors that are placed inside of the notches on the covers (Fig. 6). With this design, the absolute cover position can be determined and the spectrometer is reliably protected. 


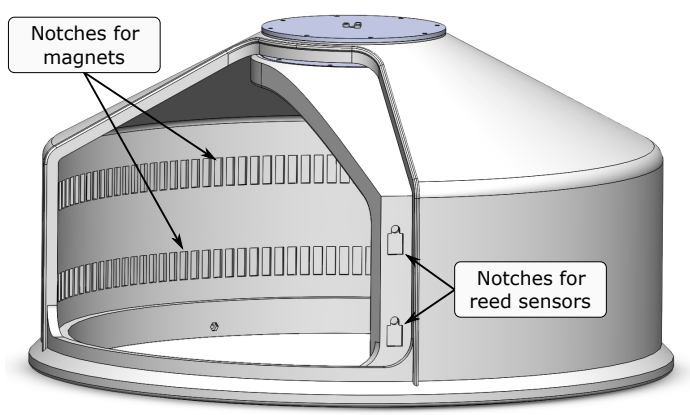

Figure 6. The image shows the cover with notches for magnets and reed sensors to track the outer cover's orientation.

Three reed sensors are glued into the notches of the inner cover, and each of the sensors has an electrical contact that closes if the sensor is exposed to a magnetic field. Nineteen neodymium magnets with a magnetic flux of $1.17 \mathrm{~T}$ each were distributed among the notches of the outer cover. One reed sensor and a single magnet positioned in the upper notches are used to detect the closed position (absolute zero position). From then on, the motion direction is determined and the number of magnets are counted. The position can be determined with a precision of about $10^{\circ}$.

To assess the distance and direction of any movement, two more reed sensors are installed next to each other in the lower notch of the inner cover. Whenever a magnet passes by the sensors, one sensor will act a little earlier than the other. This delayed operation can be used to determine the direction of movement. Multiple magnets are distributed with alternating polarity around the outer cover opposite the two sensors. Alternating the magnetic polarity creates a very weak field between two magnets as shown in Fig. 7b. If the magnets were placed with the same polarity, the magnetic field strength would not vary significantly among the magnets as depicted in Fig. 7a. Since the reed switches are sensitive to the magnetic field strength and not the polarity, this alternating arrangement ensures the sensors operate reliably.

To make sure that the sun path to the tracking mirrors is never blocked, the solar-tracking mirrors' azimuthal orientation is read directly from the mirror control. The corresponding position for the outer cover is determined and set as the target position for the cover's control unit.

\subsection{Thermal regulation}

There are several thermal concerns regarding the EM27/SUN. For instance, the solar tracker's stepping motors may stall in freezing temperatures, possibly due to greasing. Another point is the unknown impact of heat on the system. Inside the EM27/SUN, an InGaAs detector senses the intensity of the NIR light coming out of the interferometer. Typical InGaAs detectors have a temperature-dependent transfer function (Hamamatsu Photonics K. K., 2015), which means they function best with a constant operating
Table 2. The table shows the expected maximum power dissipation of all components inside the enclosure.

\begin{tabular}{lr}
\hline Component & Max. power \\
\hline Spectrometer with solar tracker & $45 \mathrm{~W}$ \\
Laptop & $30 \mathrm{~W}$ \\
Power supplies and UPS & $35 \mathrm{~W}$ \\
Router, voltage converters, etc. & $\sim 10 \mathrm{~W}$ \\
Total & $\sim 120 \mathrm{~W}$ \\
\hline
\end{tabular}

temperature. Further, condensation needs to be prevented. When warm air hits cold equipment, water vapor in the air will condensate on the cold surfaces of the equipment and tracking mirrors, which can distort the data, damage the electronics, or even potentially cause mirror degradations. A ventilation system combined with an electric heater was considered as a solution, but this has several downsides. First of all, high humidity may be vented into the enclosure, necessitating an air dehumidification system. Furthermore, a number of openings would be necessary for the ventilation, increasing the risk of rainwater leakage and that small animals could enter the enclosure. Finally, much heat may escape driven by wind blowing through ventilation openings when heating is needed.

Thus, another much easier approach that even offers cooling below the outside temperature was chosen. A thermoelectric cooler (TEC), using a solid-state Peltier device, was installed at one of the walls of the base cabinet. The TEC element is controlled by electrical current and transfers heat energy into or out of the enclosure. It does not require holes for ventilation and therefore keeps the enclosure waterproof as well as closed to animals. Further, by maintaining the temperature inside the enclosure between 24 and $25^{\circ} \mathrm{C}$, condensation can be avoided.

To prevent an unintended heat exchange between the inside and outside of the enclosure, thermal insulation is installed. A $13 \mathrm{~mm}$ layer of special foam with very low thermal conductivity, less than $0.040 \mathrm{Wm}^{-1} \mathrm{~K}^{-1}$, covers the inside of the base cabinet, allowing low heat transport even on high thermal gradients between the inside and the outside.

Because the enclosure houses many heat sources, cooling represents a greater challenge than heating. The power necessary for cooling is calculated by the given values of the components' specifications in their data sheets. A maximum power of about $120 \mathrm{~W}$ (see Table 2) must be transferred out of the enclosure. The specification of the selected TEC lists a cooling capacity of $135 \mathrm{~W}$. Thus, about $15 \mathrm{~W}$ of extra cooling power will be available. Eq. (1) is a rough estimate of the thermal diffusion. It shows that the cooling system can sufficiently cool the inside to about $2 \mathrm{~K}$ below the outside temperature.

$$
\Delta T=\frac{\dot{Q} l}{\lambda A}=\frac{15 \mathrm{~W} \cdot 13 \mathrm{~mm}}{0.040 \mathrm{Wm}^{-1} \mathrm{~K}^{-1} \cdot 2.48 \mathrm{~m}^{2}} \approx 1.97 \mathrm{~K},
$$




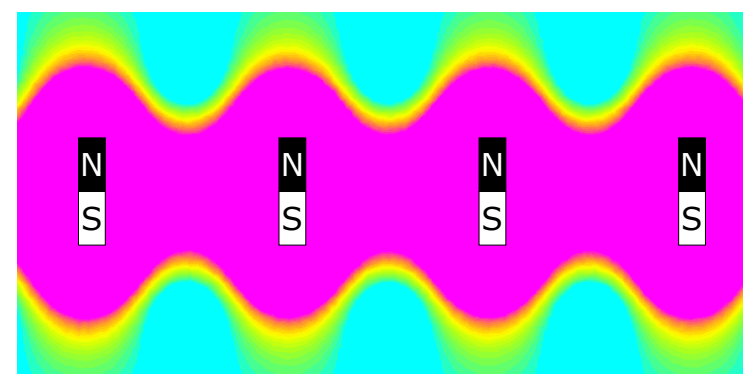

(a)

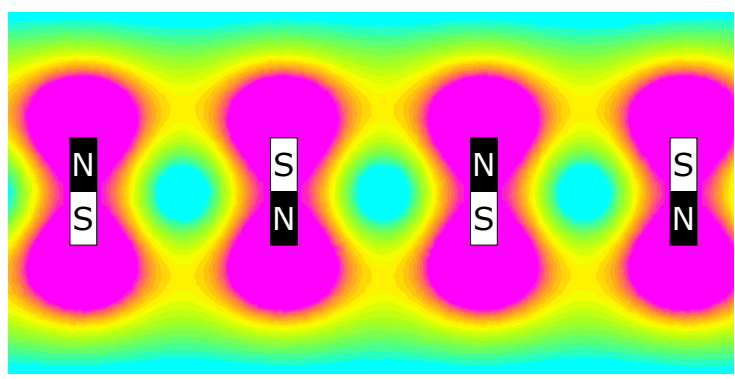

(b)

Figure 7. Comparison of the magnetic field strength when placing the magnets of the same polarity (a) and alternating polarity (b). Cyan represents a weaker magnetic field, whereas pink represents a stronger field.

where $\dot{Q}$ stands for the thermal power to be transported along the distance $l$. $A$ is the area of the thermal conduction and $\lambda$ is the thermal conductivity of the material within the volume $A \cdot l$.

\subsection{Rain sensor}

A Hydreon RG-11 optical rain sensor is installed on top of the enclosure next to the cover. When rain is detected, the integrated logic of the enclosure will automatically close the cover and send a message to the measurement system. This message may stop ongoing measurements or inform an administrator.

The sensor works as follows: an infrared LED emits light into a dome-shaped lens. The light is reflected by the lens surface and travels along its shape to the other side where it strikes a photodiode, which monitors the light intensity. When a water drop lands on the surface of the lens, less light will be detected by the photodiode because the drop allows the light to escape by refraction. RG-11 was chosen due to its dome-shaped surface. It is an improvement over a flat surface as the drops do not accumulate on the surface, which could produce a false signal after the rain stops.

\subsection{Gas spring}

As described in Sect. 2.1, the enclosure's design is based on a control cabinet. In an upright position, the cabinet's door can easily be accessed and will stay in any position the user leaves it. Hence, it is not equipped with any door openers or springs. In this application, the cabinet's orientation is changed, so the door is now located on top. Therefore, the door's open positions are no longer stable and gravity will always push the door down. Hence, a gas spring has been added to the door.

A very simplified model, shown in Fig. 8, is used to properly position and dimension the gas spring. The gas spring is designed such that it does not close on its own at any position. Thus, an operator can never be injured by the door dropping. The damping of the gas spring is very strong, so that any movement is curbed to very slow speed. Additional

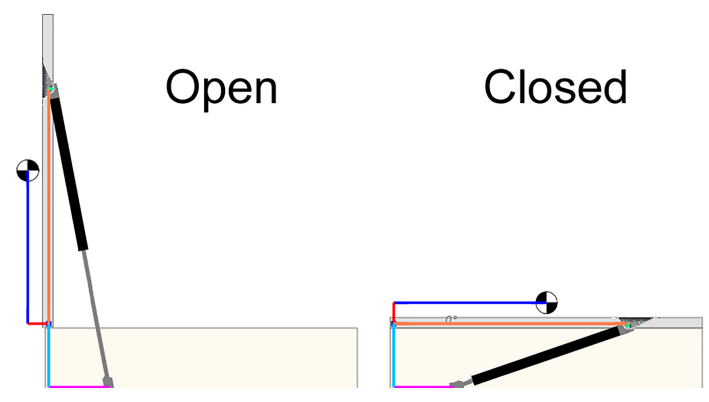

Figure 8. Simplified model of the base cabinet and its door to calculate the joint positions and the strength of the gas spring.

damping at the end positions ensures extra low acceleration, so that the cover will not be subject to strong impacts at those positions. The gas spring has a length of $582 \mathrm{~mm}$ and a maximum travel of $250 \mathrm{~mm}$. Its pushing force is about $180 \mathrm{~N}$, which is sufficient to lift the $15 \mathrm{~kg}$ door. The maximum force at the joints is calculated as $270 \mathrm{~N}$.

\subsection{Uninterruptible power supply}

As previously mentioned, an electric motor rotates the outer cover. There is no mechanical fallback that can close the cover in case of a power outage. Hence, an uninterruptible power supply (UPS) is mandatory. Additionally, temporary power outages could erase measurements and place the system in an undefined state; therefore, the UPS is designed to supply sufficient energy to the measurement system for several minutes.

\subsection{Relays}

While operating the EM27/SUN in the past, occasionally unexpected errors occurred, some of which could only be resolved by restarting the spectrometer or its camera. Thus, two relays are included so that a remote operator or controlling software can switch off the power to the spectrometer and disconnect its USB camera from the laptop. 


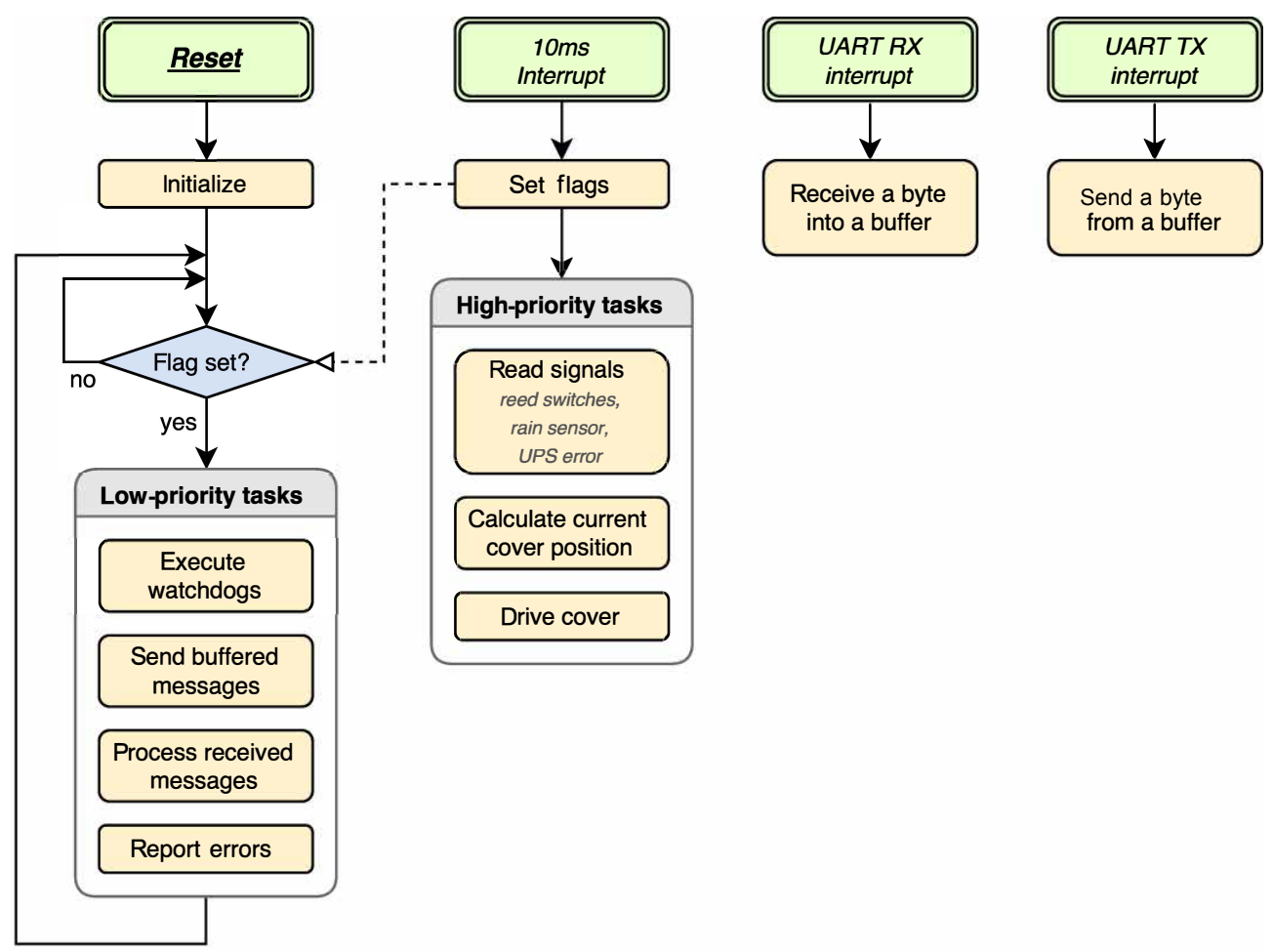

Figure 9. The flow chart visualizes the structure of the microcontroller's software.

The first relay is mounted on the DIN rail and connects the power to the EM27/SUN. Besides resolving errors, this switch allows powering the instrument off at night and thus helps to save energy. The second relay is located on a USB intermediate plug. It was developed to enable the solar tracker's USB camera to be disconnected. In the past, the only effective solution to recover from camera errors was to physically reconnect the camera. Therefore, the relay on the intermediate plug simply disconnects the $5 \mathrm{~V}$ wire of the USB connection, which simulates the physical unplugging of the USB connector. As long as the relay is closed, the USB data communication will not be affected, since the data lines of the USB connection remain untouched. With the help of this circuitry, the USB camera can be reset by a remote operator or automatically by a software.

\subsection{Enclosure control board}

The control board of the enclosure handles low-level access to the enclosure's hardware. It provides protective safety features, like closing the cover whenever an error occurs. Furthermore, it receives commands from the laptop and operates the enclosure hardware accordingly. The control board works independently to guarantee a very high level of fail safety. Almost every signal on and off the board is designed for the highest possible fault tolerance to ensure reliable operation in every situation.
The control board implements the most critical safety features and serves as a tool for the laptop to operate the enclosure. The central brain of the board is an Atmel ATmega168 microcontroller. The system on chip (SOC), which includes a small, $20 \mathrm{MHz}$ RISC (reduced instruction set computer) processor with integrated memory, is very robust. Besides a crystal and some capacitors, no external hardware is required, which makes the control board very reliable. For the communication with the laptop, an FTDI FT232RL USB to RS232 converter is placed on the board. More details on the operation of the enclosure control board and the communication interface are given in Sect. 3.1.

\section{Software}

\subsection{Enclosure control board software}

The enclosure control board is a central component of the enclosure. The software for the microcontroller on the board implements the enclosure's basic logic, controlling the electric motor of the cover and reading its position by evaluating the signals of the sensors in the cover. Moreover, it receives rain sensor and UPS signals and communicates with the measurement system via USB. Controlling some relays, the enclosure control board can even turn the spectrometer on or off inside the enclosure. Its design, with self-monitoring and a fail-safe circuit, guarantee high reliability and security, en- 


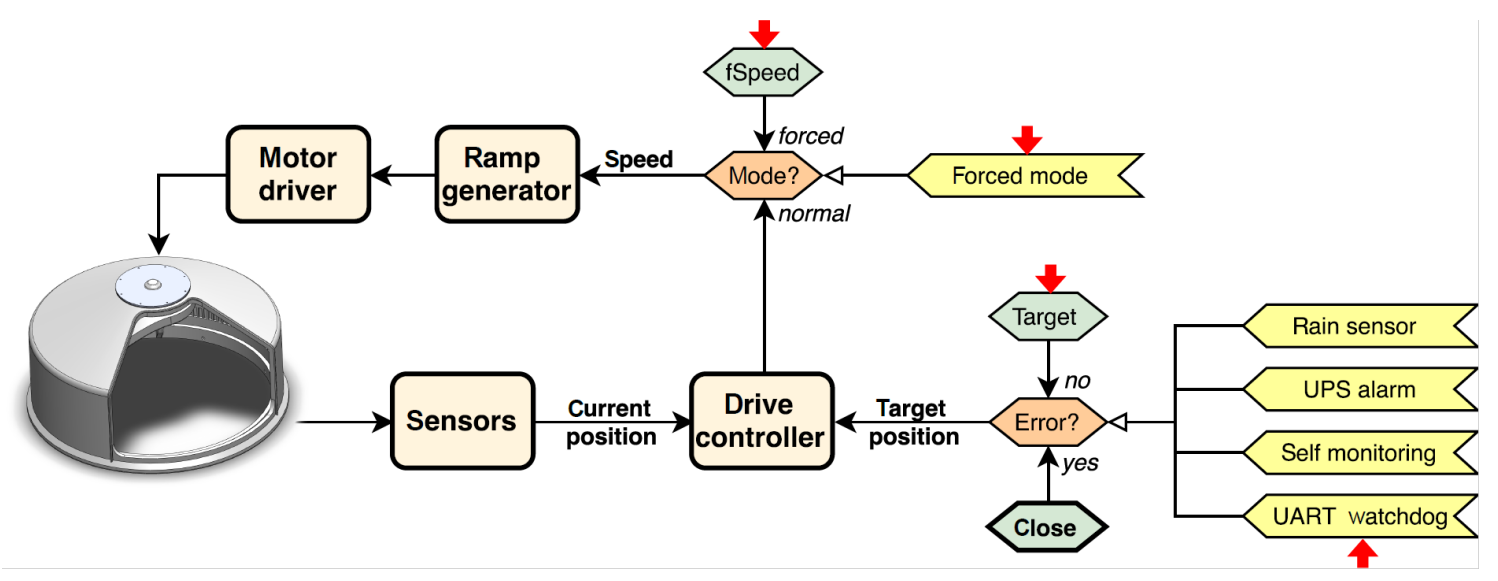

Figure 10. The flow chart shows the design of the cover control flow. The red arrows indicate values that can be changed or influenced by the user via the USB interface.

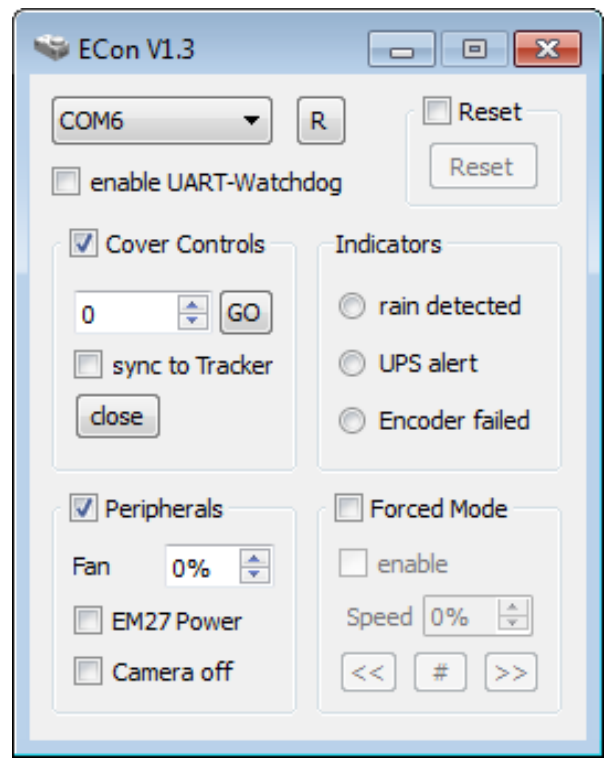

Figure 11. The image shows the graphical user interface called ECon. With the given controls and indicators, nearly every enclosure function can be controlled and observed.

suring full control and the highest protection of the instrument inside.

\subsubsection{Structure of the software}

To exploit the limited hardware resources as much as possible, the software is split into a set of high- and low-priority tasks. The most important high-priority task is the control of the cover as explained in Sect. 3.1.2. The low-priority tasks are the less time-critical communication processes. Resetting the hardware watchdog is also classified as low priority.

Figure 9 shows a full overview of the program flow. The reset node is the starting point of the program after every reset. After that, the processor initializes and enters its main

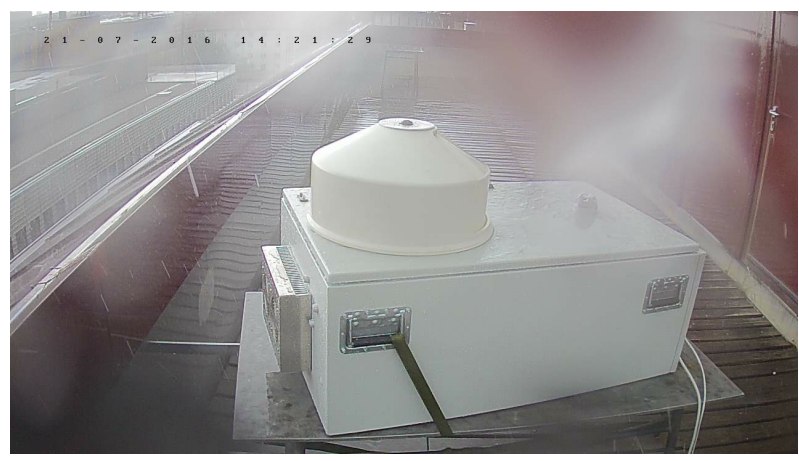

Figure 12. Example of the bad weather the fully equipped enclosure was exposed to.

loop, where it executes all the low-priority tasks. An interrupt will be executed every $10 \mathrm{~min}$, processing the high-priority tasks.

\subsubsection{Cover control scheme}

Figure 10 depicts the cover control flow. While operating in normal mode, the drive controller compares the current position to a target value, which can be derived from the azimuthal orientation of the tracking mirrors or the closed position. Depending on the distance between the two orientations, a target speed is calculated and fed into a ramp generator that slowly accelerates or decelerates the rotation of the cover to reduce mechanical stress. Its output signal controls the motor driver. The current position of the cover is calculated from the sensor readings and fed back to the drive controller. In case of any error, the target position will be set to the closed position, as explained above.

An additional forced mode is included to allow full control in case of emergency. If an error occurs, the loop can be cut open via the interface, and a fixed value can be fed into the ramp generator. Hence, a remote operator can take full 
(a)

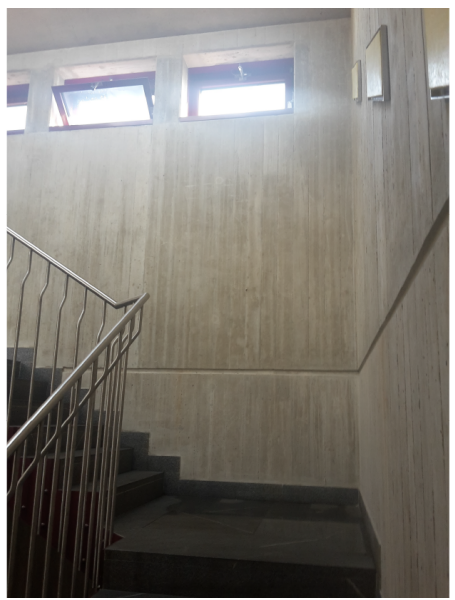

(b)

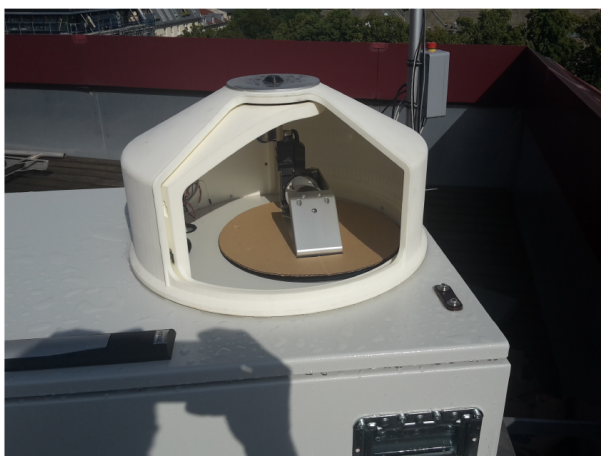

Figure 13. (a) Image of flooded staircase. The water was blown into the tilted window. (b) Image of the open enclosure after the storm. As can be seen, no water was able to penetrate the cover.
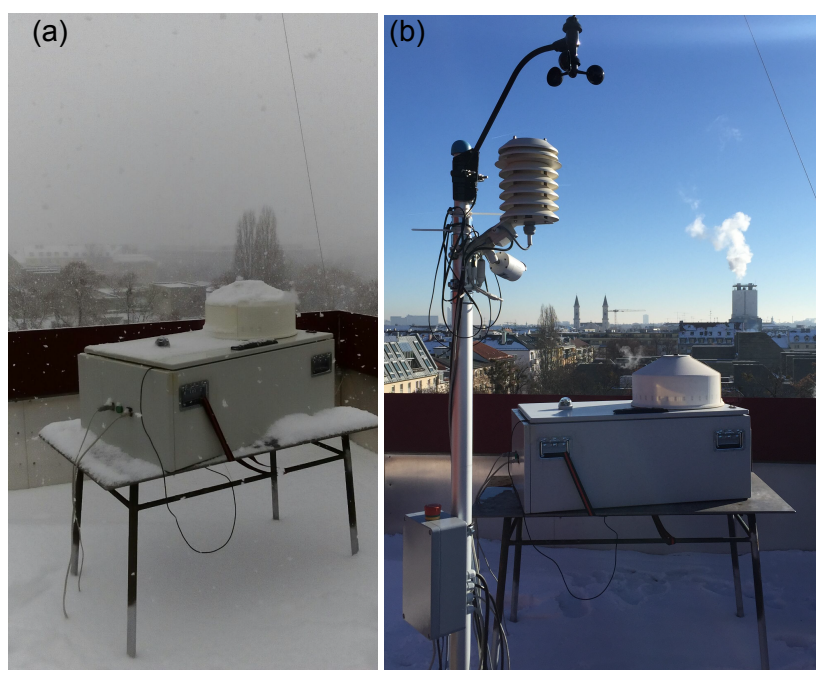

Figure 14. (a) During winter 2016, with a number of centimeters of snowfall on the enclosure with the spectrometer located inside. (b) The system taking measurements in cold winter below $0^{\circ} \mathrm{C}$.

control of the cover and navigate it into a secure position if needed.

\subsubsection{Safety features}

The microcontroller's hardware includes a watchdog feature that monitors the processor's operation. A watchdog is basically a timer that expects a reset signal in its configurable period. In normal operation the watchdog timer will be reset before a timeout occurs. However, if the processor gets hung at any position in the program, the watchdog timer will time out and consequently trigger a full reset, guaranteeing very high operational reliability.

Additionally, the software provides an optional UART watchdog. When enabled, it will expect an "I am alive" mes- sage every $5 \mathrm{~s}$. In contrast to the hardware watchdog, which is not optional, a timeout of the UART watchdog will not trigger a system reset. Instead, an error flag will be set and the program will close the cover (see Fig. 10).

\subsection{Computer software}

We also developed a computer program, written in python, to offer a set of methods to control the enclosure's different functions with a graphical user interface (GUI). The GUI is called ECon (short for Enclosure Control) and appears as a window on the desktop (Fig. 11).

\section{Results}

After constructing the enclosure, its functions were tested. First of all, the dryness inside the enclosure after extreme stormy and snowy conditions is confirmed. Secondly, the ability to regulate the temperature inside the enclosure is examined. Next, we investigate whether it blocks the sun during the course of the day. Finally, the remote controllability and operability are shown.

\subsection{Rain and snow test}

The enclosure encountered several extreme weather events. The surveillance camera, installed to allow for remote live viewing of the enclosure, captured the stormy conditions in Fig. 12. Even after these extreme rainy and windy conditions, the enclosure remained completely dry inside and not a single drop of water entered. Figure 13 shows that no water was blown into the cover. The rain sensor also reliably detected the first drop of water on its sensitive area and the cover was closed in less than $6 \mathrm{~s}$.

In winter 2016, Munich experienced a large amount of snow, and the enclosure proved to be robust against snowfall 


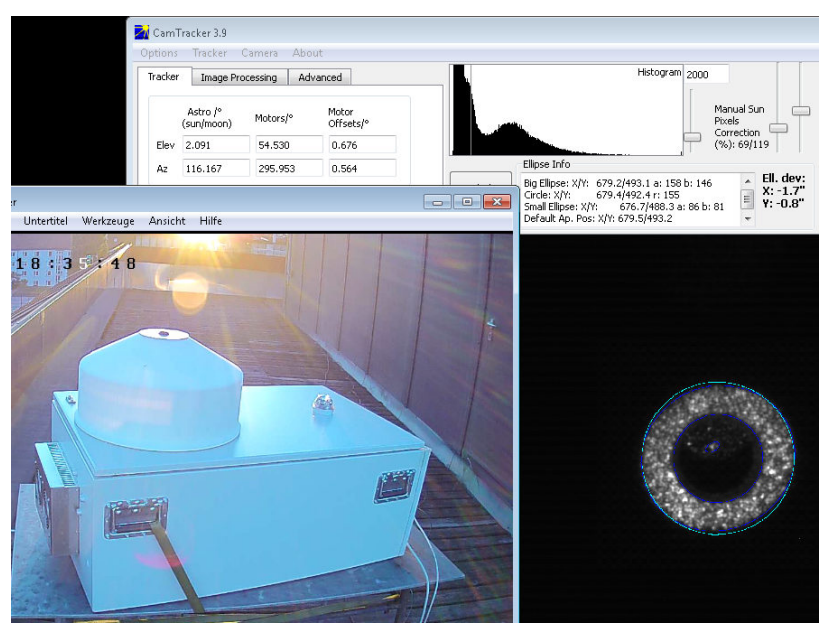

(a)
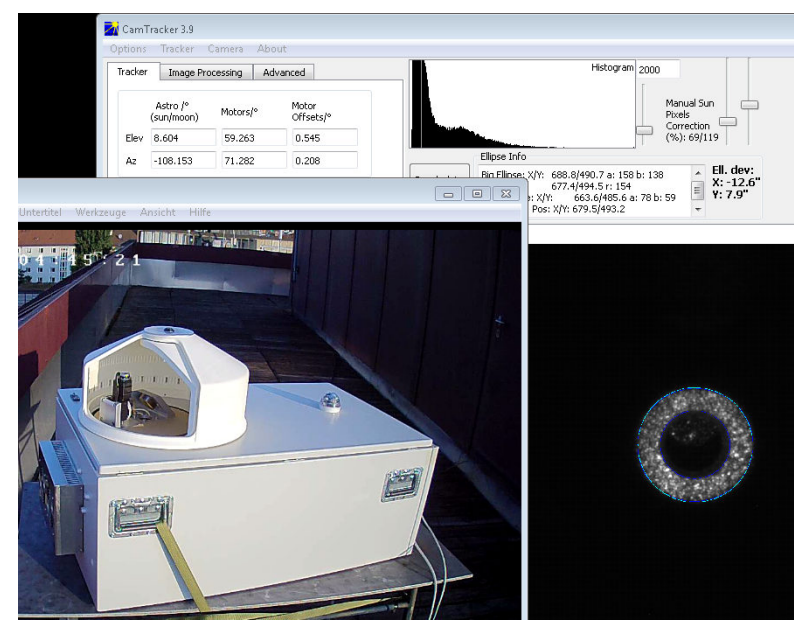

(b)

Figure 15. The images show screen shots of the laptop in the late evening (a) and early morning (b). Panel (a) shows the instrument tracking the sun at an azimuthal angle of $296^{\circ}$ on 29 July at 20:35 LT. Panel (b) shows the tracker following the sun at about $71^{\circ}$ azimuthal angle on 30 July at 06:45 LT. As expected, in both cases the cover did not block the sunlight.

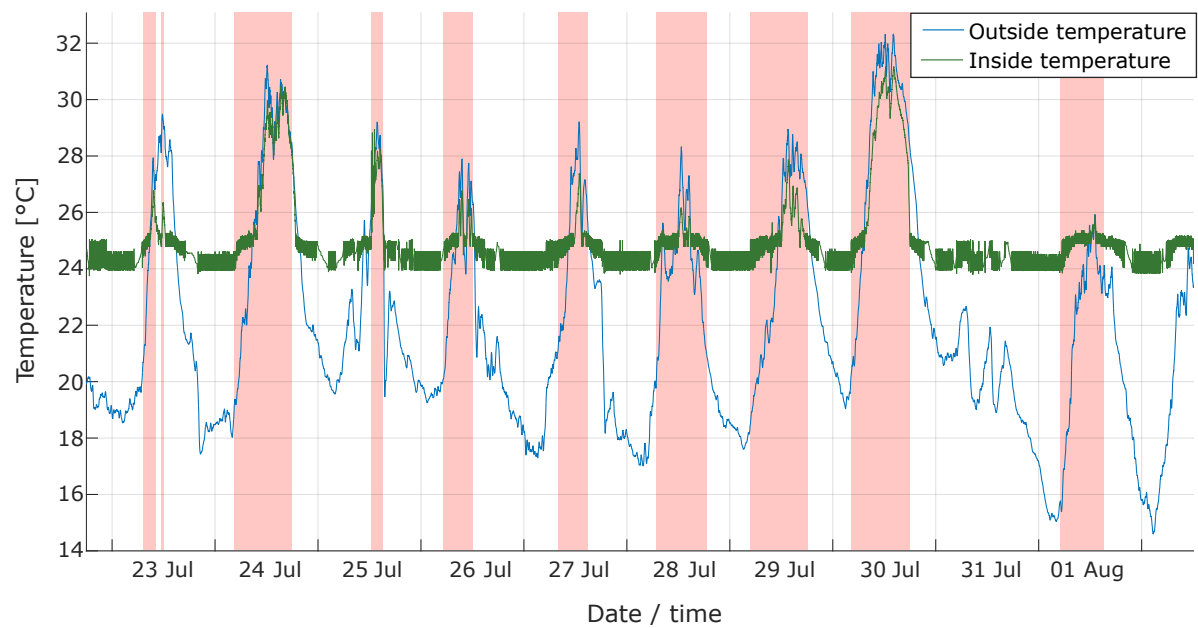

Figure 16. The diagram shows the internal and external temperature of the enclosure. The red bars illustrate time periods during which the measurement system was active and therefore produced heat that had to be pumped out of the enclosure by the TEC.

on the cover (Fig. 14). The enclosure is constantly heated and the spectrometer is able to carry out measurements whenever it is sunny, even if the ambient temperature falls below $0^{\circ} \mathrm{C}$. In addition, no freezing occurred. The enclosure is heated to $25^{\circ} \mathrm{C}$. The warm air rises up and prevents freezing of trapped water.

\subsection{Unblocked sunlight}

During the 1.5-year test period, the enclosure showed no single case of blocking the sun during measurements. The test azimuthal angle ranges from $71^{\circ}$ to about $296^{\circ}$ (see Fig. 15). On 29 July 2016, the latter angle was checked in the evening. As shown in Fig. 15a, the sun was not blocked at the $296^{\circ}$ azimuthal angle ( $88^{\circ}$ zenith). Afterwards, the sun disappeared behind clouds and buildings. Optical investigations revealed that the sun remained unblocked until close to $300^{\circ}$. On the next day, the sun was unblocked at an azimuthal angle of about $71^{\circ}$ in the morning ( $82^{\circ}$ zenith), as shown in Fig. 15 b.

\subsection{Thermal regulation}

The enclosure's thermal regulation was tested by logging thermal readings of the outside and inside temperatures during normal operation (see Fig. 16). If the temperature falls below the configured threshold of $24^{\circ} \mathrm{C}$, the TEC is turned on for heating until the temperature rises above $24.1^{\circ} \mathrm{C}$. The same is valid for cooling, whereby the TEC is powered for cooling at above $25^{\circ} \mathrm{C}$ and disabled at $24.9^{\circ} \mathrm{C}$. The regulator has only three states: heating, cooling, and off. There is 


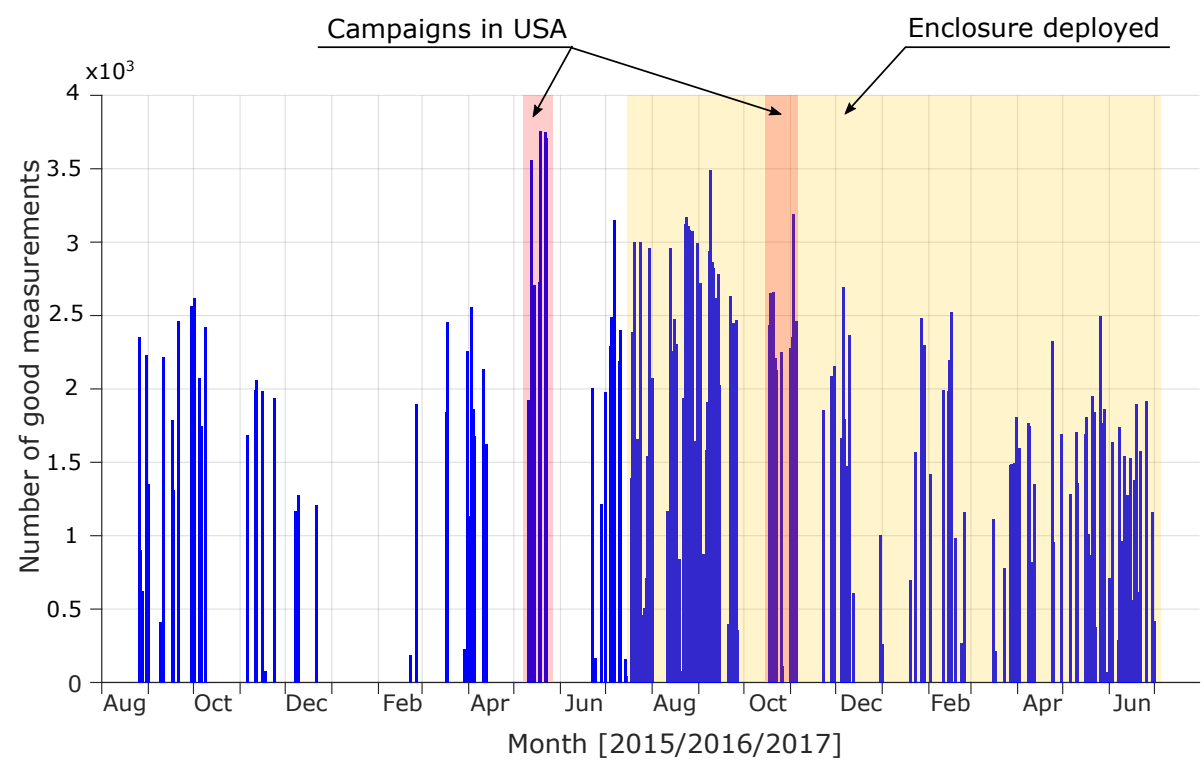

Figure 17. Visualization of the amount of daily sampled data with the EM27/SUN. The yellow region marks the time when the EM27/SUN was deployed inside of the enclosure. During the time periods indicated by the red regions, the EM27/SUN participated in measurement campaigns, first in Indianapolis and then in the San Francisco Bay Area, USA, respectively. Due to the shipping time to and back from the USA, measurements were not taken a month before and after the campaigns.

no intermediate state, which would allow for a smoother regulation. Thanks to the temperature control, no condensation was observed on the mirrors or other components inside the base cabinet.

During construction, the TEC was designed to pump out the heat of the measurement system. Cooling below the outside temperature were considered a useful feature after the enclosure was assembled. The built-in reserves of the TEC are not large enough to cool the system more than just a few degrees below the outside temperature (see Fig. 16). On 23 July 2016 the temperature was controlled within the desired range as long as the outside temperature was lower than $27^{\circ} \mathrm{C}$ or the measurement system was turned off, so that less heat is generated.

\subsection{Remote operability}

The measurement system can be fully remotely operated. Using remote desktop software, any operator can log in from any computer and take control of the system. Because of the enclosure no physical attendance is required to start, stop, or monitor measurements. Figure 17 shows the amount of data collected daily. As can be seen, the density increased clearly when the enclosure was set up for operation. Measurements were taken nearly every day; even small windows in the clouds were used to sample the data.

\section{Future work}

In future, rain prediction information can be integrated to assist in the decision making regarding cover control. One potential approach could be to assess real-time online rain radar data and meteorological forecasts to predict upcoming weather.

Further improvements of the enclosure could focus on a localized thermal regulation. One could specify the most sensitive components of the measurement system and pay particular attention to their thermal regulation, which could also meet the thermal demands while reducing the total energy consumption.

Another enclosure improvement could target a lightweight and portable version, which would be better suited for mobile measurements as needed in campaigns. The current enclosure was designed for stationary use and gives proof of concept. Nevertheless, there is high demand to use the enclosure for campaigns. This raises a whole new set of requirements such as reduced weight, size, and power consumption. 


\section{Conclusions}

We designed, engineered, and assembled an automated enclosure for an atmospheric solar-tracking instrument, the EM27/SUN, and successfully tested it under extreme weather conditions. The automated solar-tracking system is located in central Munich $\left(48.15^{\circ} \mathrm{N}, 11.57^{\circ} \mathrm{E}\right)$.

The enclosure is suited for fully autonomous operation and enables easy handling of the measurement system. When potentially poor weather conditions arise, the system can be shut down and protected within seconds. In case of sudden rain, the enclosure reliably protects the measurement system by closing its cover. In this manner, the measurement system is sheltered from heavy storms, rainfall, and snowfall without the need for any physical human interaction.

Remote access can be obtained by any smartphone or computer. Thus, the system can be observed and controlled from anywhere in the world. This considerably reduces the inhibition level to start measurements, resulting in a significant increase in the amount of data collected. Furthermore, the automated enclosure reduces the need for costly human effort and optimizes the chance to sample good data, even during periods of unstable weather conditions. Accordingly, the measurement system is highly efficient, which should increase the significance of any scientific outcome derived from these data.

In sum, the enclosure allows for a fully automated measurement system, guaranteeing a maximum amount of data collection while minimizing the operational risks and costs, thus providing a foundation for long-term GHG monitoring sensor network.

Data availability. All data are included in the paper. Inquiries about additional data can be directed to the corresponding author, Jia Chen (jia.chen@tum.de).

Competing interests. The authors declare that they have no conflict of interest.

Acknowledgements. We gratefully thank Gerold Wunsch for helping with the cover design and Florian Dietrich for further developing and operating the automatic system. We also acknowledge our Bruker colleagues Peter Maas and Gregor Surawicz for technical support. Further, we thank Frank Hase, Bruce Daube, Steven C. Wofsy, Andreas Meichelböck, Johannes C. Paetzold, Duc Hai Nguyen, and Patrick Aigner for fruitful discussions and Stephen Starck from the TUM language center for the helpful English editing. Jia Chen is supported by Technische Universität München - Institute for Advanced Study, funded by the German Excellence Initiative and the European Union Seventh Framework Programme under grant agreement no. 291763.

This work was supported by the German Research
Foundation (DFG) and the Technische Universität

München within the funding programme

Open Access Publishing.

Edited by: Frank Hase

Reviewed by: two anonymous referees

\section{References}

Bruker Optic GmbH: IFS 125 User Manual, available at: http: //spec.jpl.nasa.gov/ftp/pub/outgoing/IFS125HR_manual.pdf (25 March 2018), 2006.

Butz, A., Dinger, A. S., Bobrowski, N., Kostinek, J., Fieber, L., Fischerkeller, C., Giuffrida, G. B., Hase, F., Klappenbach, F., Kuhn, J., Lübcke, P., Tirpitz, L., and Tu, Q.: Remote sensing of volcanic $\mathrm{CO}_{2}, \mathrm{HF}, \mathrm{HCl}, \mathrm{SO}_{2}$, and $\mathrm{BrO}$ in the downwind plume of $\mathrm{Mt}$. Etna, Atmos. Meas. Tech., 10, 1-14, https://doi.org/10.5194/amt10-1-2017, 2017.

Chen, J., Viatte, C., Hedelius, J. K., Jones, T., Franklin, J. E., Parker, H., Gottlieb, E. W., Wennberg, P. O., Dubey, M. K., and Wofsy, S. C.: Differential column measurements using compact solartracking spectrometers, Atmos. Chem. Phys., 16, 8479-8498, https://doi.org/10.5194/acp-16-8479-2016, 2016.

Chen, J., Nguyen, H., Toja-Silva, F., Heinle, L., Hase, F., and Butz, A.: Power Plant Emission Monitoring in Munich Using Differential Column Measurements, in: EGU General Assembly Conference Abstracts, 19, 16423, Vienna, Austria, 23-28 April 2017.

Chen, J., Dietrich, F., Franklin, J., Jones, T., Butz, A., Luther, A., Kleinschek, R., Hase, F., Wenig, M., Ye, S., Nouri, A., Frey, M., Knote, C., Alberti, C., and Wofsy, S.: Mesoscale Column Network for Assessing GHG and NOx Emissions in Munich, in: EGU General Assembly Conference Abstracts, 20, 10192, Vienna, Austria, 8-13 April 2018.

Franklin, J. E., Jones, T. S., Chen, J., Parker, H., Hedelius, J., Wennberg, P., Dubey, M. K., Cohen, R. C., Guha, A., Sargent, M., Davis, K. J., Mielke, L., Fischer, M., and Wofsy, S.: A three-dimensional observation network for determining urban emissions of $\mathrm{CO}_{2}$ and $\mathrm{CH}_{4}$, in 2017 North American Carbon Program, North Bethesda, MD, USA, 2017, available at: https://www.nacarbon.org/meeting_2017/abs_and_ discussions/mtg2017_ab_searchab_id161.html (last access: 23 March 2018), 2017.

Frey, M., Hase, F., Blumenstock, T., Groß, J., Kiel, M., Mengistu Tsidu, G., Schäfer, K., Sha, M. K., and Orphal, J.: Calibration and instrumental line shape characterization of a set of portable FTIR spectrometers for detecting greenhouse gas emissions, Atmos. Meas. Tech., 8, 3047-3057, https://doi.org/10.5194/amt-83047-2015, 2015.

Geibel, M. C., Gerbig, C., and Feist, D. G.: A new fully automated FTIR system for total column measurements of greenhouse gases, Atmos. Meas. Tech., 3, 1363-1375, https://doi.org/10.5194/amt-3-1363-2010, 2010.

Gisi, M., Hase, F., Dohe, S., and Blumenstock, T.: Camtracker: a new camera controlled high precision solar tracker system for FTIR-spectrometers, Atmos. Meas. Tech., 4, 47-54, https://doi.org/10.5194/amt-4-47-2011, 2011. 
Gisi, M., Hase, F., Dohe, S., Blumenstock, T., Simon, A., and Keens, A.: $\mathrm{XCO}_{2}$-measurements with a tabletop FTS using solar absorption spectroscopy, Atmos. Meas. Tech., 5, 2969-2980, https://doi.org/10.5194/amt-5-2969-2012, 2012.

Hamamatsu Photonics K. K.: InGaAs Photodiodes, available at: https://www.hamamatsu.com/resources/pdf/ssd/ingaas_ kird0005e.pdf (25 March 2018), 2015.

Hase, F.: Improved instrumental line shape monitoring for the ground-based, high-resolution FTIR spectrometers of the Network for the Detection of Atmospheric Composition Change, Atmos. Meas. Tech., 5, 603-610, https://doi.org/10.5194/amt-5603-2012, 2012.

Hase, F., Frey, M., Blumenstock, T., Groß, J., Kiel, M., Kohlhepp, R., Mengistu Tsidu, G., Schäfer, K., Sha, M. K., and Orphal, J.: Application of portable FTIR spectrometers for detecting greenhouse gas emissions of the major city Berlin, Atmos. Meas. Tech., 8, 3059-3068, https://doi.org/10.5194/amt-8-3059-2015, 2015.

Hase, F., Frey, M., Kiel, M., Blumenstock, T., Harig, R., Keens, A., and Orphal, J.: Addition of a channel for XCO observations to a portable FTIR spectrometer for greenhouse gas measurements, Atmos. Meas. Tech., 9, 2303-2313, https://doi.org/10.5194/amt9-2303-2016, 2016.

Hedelius, J. K., Viatte, C., Wunch, D., Roehl, C. M., Toon, G. C., Chen, J., Jones, T., Wofsy, S. C., Franklin, J. E., Parker, H., Dubey, M. K., and Wennberg, P. O.: Assessment of errors and biases in retrievals of $\mathrm{X}_{\mathrm{CO}_{2}}, \mathrm{X}_{\mathrm{CH}_{4}}, \mathrm{X}_{\mathrm{CO}}$, and $\mathrm{X}_{\mathrm{N}_{2} \mathrm{O}}$ from a $0.5 \mathrm{~cm}^{-1}$ resolution solar-viewing spectrometer, Atmos. Meas. Tech., 9, 3527-3546, https://doi.org/10.5194/amt-9-3527-2016, 2016.
Klappenbach, F., Bertleff, M., Kostinek, J., Hase, F., Blumenstock, T., Agusti-Panareda, A., Razinger, M., and Butz, A.: Accurate mobile remote sensing of $\mathrm{XCO}_{2}$ and $\mathrm{XCH}_{4}$ latitudinal transects from aboard a research vessel, Atmos. Meas. Tech., 8, 50235038, https://doi.org/10.5194/amt-8-5023-2015, 2015.

Neefs, E., De Mazière, M., Scolas, F., Hermans, C., and Hawat, T.: BARCOS, an automation and remote control system for atmospheric observations with a Bruker interferometer, Rev. Sci. Instrum., 78, 035109, https://doi.org/10.1063/1.2437144, 2007.

Sha, M. K.: Glass dome selection as a cover for protecting remote sensing instruments performing atmospheric measurements of trace gases, Technical notes, Karlsruher Institut für Technologie (KIT), Karlsruhe, 2015.

Toja-Silva, F., Chen, J., Hachinger, S., and Hase, F.: CFD simulation of $\mathrm{CO}_{2}$ dispersion from urban thermal power plant: Analysis of turbulent Schmidt number and comparison with Gaussian plume model and measurements, J. Wind Eng. Ind. Aerod., 169, 177193, https://doi.org/10.1016/j.jweia.2017.07.015, 2017.

Viatte, C., Lauvaux, T., Hedelius, J. K., Parker, H., Chen, J., Jones, T., Franklin, J. E., Deng, A. J., Gaudet, B., Verhulst, K., Duren, R., Wunch, D., Roehl, C., Dubey, M. K., Wofsy, S., and Wennberg, P. O.: Methane emissions from dairies in the Los Angeles Basin, Atmos. Chem. Phys., 17, 7509-7528, https://doi.org/10.5194/acp-17-7509-2017, 2017.

Wunch, D., Toon, G. C., Blavier, J. F., Washenfelder, R. A., Notholt, J., Connor, B. J., Griffith, D. W., Sherlock, V., and Wennberg, P. O.: The total carbon column observing network, Philos. T. Roy. Soc. A, 369, 2087-2112, 2011. 